\title{
Special Energies and Special Frequencies
}

\author{
M. Endrullis and H. Englisch \\ Sektion Mathematik, Karl-Marx-Universität Leipzig, DDR-7010 Leipzig, \\ German Democratic Republic
}

\begin{abstract}
Special frequencies" have been asserted to be zeros of the density of frequencies corresponding to a random chain of coupled oscillators. Our investigation includes both this model and the random one-dimensional Schrödinger operator describing an alloy or its discrete analogue. Using the phase method we exactly determine a bilateral Lifšic asymptotic of the integrated density of states $k(E)$ at special energies $E_{s}$, which is not only of the classical type $\exp \left(-c /\left|E-E_{s}\right|^{1 / 2}\right)$ but also $\exp \left(-c^{\prime} /\left|E-E_{s}\right|\right)$ is a typical behaviour. In addition, other asymptotics occur, e.g. $\left|E-E_{s}\right|^{c \prime \prime}$, which show that $k(E)$ need not be $C^{\infty}$.
\end{abstract}

\section{Introduction}

In this paper, we consider the random Schrödinger operator (Hamiltonian)

$$
H^{\omega}=-\frac{d^{2}}{d x^{2}}+V^{\omega}(x) \quad \text { on } \quad L^{2}(R)
$$

with

$$
V^{\omega}(x)=\sum_{n \in Z} V_{\omega(n)}(x-n),
$$

where the indices $\omega(n)$ are random variables on the realization space $\Omega$ with values in the set $\{1,2, \ldots, r\}$. We deal with the case in which the random process $\omega(n)$ is independent, identically distributed and the functions $V_{i}$ are form-bounded with respect to $-d^{2} / d x^{2}$ (e.g., they can be bounded or $\delta$-functions, cf. [1]) and satisfy $\operatorname{supp} V_{i} \subseteq[0,1)$ for all $i \in\{1,2, \ldots, r\}$ (the random process can be chosen more generally, cf. [2]). The operator $H^{\omega}$ defined in this way describes a one-dimensional $r$-ary random alloy in the one-body approximation.

Our interest is directed to the integrated density of states, i.e. to the limit

$$
k(E)=\lim _{L \rightarrow \infty} \frac{1}{L} N_{E}\left(H_{L}^{\omega}\right),
$$


where $H_{L}^{\omega}$ is the restriction of the Hamiltonian $H^{\omega}$ to $[0, L]$ with some, e.g. periodic, boundary conditions and $N_{E}\left(H_{L}^{\omega}\right)$ is the number of eigenvalues of $H_{L}^{\omega}$ below $E$ (counting multiplicity). Concerning the almost sure existence of the limit (3) and its independence of $\omega$, see [3-5]. When we define the phase $\varphi$ by

$$
\tan \varphi(x)=\frac{y(x)}{y^{\prime}(x)}, \quad 0 \leqq \varphi(0)<\pi
$$

[uniqueness by $\varphi(x)$ continuous] with $y$ satisfying $H^{\omega} y=E y$ as a differential equation, the following formula represents the "phase method" for the calculation of $k(E)$ (cf. $[5,6])$ :

$$
\left|k(E)-k\left(E^{\prime}\right)\right|=\lim _{L \rightarrow \infty} \frac{1}{L} \frac{\varphi_{E}^{\omega}(L)-\varphi_{E^{\prime}}^{\omega}(L)}{\pi}
$$

for almost every realization $\omega \in \Omega$.

Using (4), we exactly determine the asymptotic behaviour of the integrated density of states $k(E)$ at "special energies" $E_{s}$ which were supposed to be zeros of the density of states $k^{\prime}(E)=d k(E) / d E$ already twenty years ago [7]. One of the authors [8] extracted from the models considered in $[7,9-11]$ the following definition of a special energy:

Definition. Let $\Omega_{\text {per }}$ denote the set of all periodic realizations $\omega$. Then $E_{s}$ is a special energy of the Hamiltonian $H^{\omega}$ if

(i) there exists a periodic potential $V^{\omega \prime}$ such that $E_{s} \in \sigma\left(H^{\omega^{\prime}}\right)$,

(ii) for all $\omega^{\prime \prime} \in \Omega_{\mathrm{per}} \backslash\left\{\omega^{\prime}\right\}$ it holds that $E_{s}$ does not lie in the interior of the spectrum $\sigma\left(H^{\omega \prime \prime}\right)$, i.e. $E_{s} \notin \operatorname{int} \sigma\left(H^{\omega \prime \prime}\right)$.

Since for almost every $\omega$ it holds that

$$
\sigma\left(H^{\omega}\right)=\overline{\bigcup_{\omega^{\prime} \in \Omega \text { per }} \sigma\left(H^{\left(\omega^{\prime}\right)}\right.}
$$

(cf. $[12,13])$ a special energy almost surely lies in the spectrum $\sigma\left(H^{\omega}\right)$. Thereby the definition combines the special energies lying in the interior of a band with the band edges. The case that $E_{s} \in \sigma\left(H^{\omega}\right)$ for a.e. $\omega \in \Omega$, but $E_{s} \notin \sigma\left(H^{\omega \prime}\right)$ for all $\omega^{\prime} \in \Omega_{\text {per }}$, which can occur for $r=\infty$, is not included in our definition.

Underlying the definition of a special energy, in Sects. 2 and 3 we give sufficient conditions under which $k^{\prime}(E)=0$ for $E=E_{s}$. For it we work with the integrated density of states because the existence of $k^{\prime}(E)$ is not proved in general [14]. Craig and Simon [15] have only shown that $k(E)$ is log Hölder continuous.

Our results show that at special energies the asymptotic behaviour

$$
\left|k(E)-k\left(E_{s}\right)\right| \sim \exp \left(-c / \mid E-E_{s}\right)
$$

is typical, what had been stated in [8] for the first time. Parallel to us Nieuwenhuizen [16] predicted (5) up to a relative inaccuracy of $20 \%$ by computer calculations. In addition, we may have

$$
\left|k(E)-k\left(E_{s}\right)\right| \sim \exp \left(-c^{\prime} /\left|E-E_{s}\right|^{1 / 2}\right)
$$

(known as Lifšic singularity for $k^{\prime}(E)$ at band edges, cf. [17] and ref. therein) and some other asymptotics occur, e.g., in a very special case (see Sect. 3) it holds a 
power law

$$
\left|E-E_{s}\right|^{c+\delta} \leqq\left|k(E)-k\left(E_{s}\right)\right| \leqq\left|E-E_{s}\right|^{c}
$$

with some constant $c>0$ and arbitrary small $\delta>0$. In his "Fifteen Problems in Mathematical Physics," Simon [18] asks the question whether $k(E)$ is a $C^{\infty}$ function (Problem 12C). The behaviour (7) indicates that $k(E)$ need not be $C^{\infty}$ in $E_{s}$, since $c$ can take arbitrary values of $(0,1)$ in dependence on the potentials $V_{j}$ and their probability distribution. The asymptotic of type 4 introduced in Sect. 3 indicates the same. ${ }^{1}$

All our considerations also apply to the discrete analogue of (1) for which Wegner [19] showed that $k^{\prime}(E)$ is strictly positive for a large class of random potentials with continuous distribution. Thus, our assumption of a discrete distribution seems to be essential. Nevertheless, there are models with continuous potential distribution and vanishing density of states at special energies as numerical calculations of Nieuwenhuizen [16] done for the Kronig-Penney model with Poisson-distributed strength of the $\delta$-potential show.

Our investigation also includes the chain of coupled vibrating oscillators described by the operator

$$
\left(h^{\omega} y\right)_{n}=\frac{1}{m_{\omega(n)}}\left(-y_{n+1}-y_{n-1}+2 y_{n}\right), \quad n \in Z,
$$

with independent, identically distributed random masses $m_{\omega(n)} \in\left\{m_{1}, m_{2}, \ldots, m_{r}\right\}$. Thereby, in connection with this model, since it describes the phonon evolution in harmonic crystals, it is usual to speak about the integrated density of squared frequencies $f\left(v^{2}\right)$, which is defined in complete analogy to $k(E)$.

Historically, the investigations just started with the disordered diatomic (i.e. $r=2$ ) chain (8), stimulated by Dean's computer calculations [9] which showed a very fine structure of $f^{\prime}\left(v^{2}\right)$ with several sharp peaks and some frequencies $v_{s}$ at which $f^{\prime}\left(v^{2}\right)=0$. Trying to give a theoretical explanation for these zeros, Matsuda [7] introduced for them the notation "special frequencies." But his paper as well as the works by Hori and Matsuda [10] or Hori [11] do not answer the question whether indeed $f^{\prime}\left(v_{s}^{2}\right)=0$. By a plausibility argument (cf. [11] and see [8] for its discussion), it was only stated that $\lim _{v \rightarrow v_{s}-0} f^{\prime}\left(v^{2}\right)=0$. (For further references see
[20]; cf. also [21].)

Our considerations show that the density of squared frequencies $f^{\prime}\left(v^{2}\right)$ indeed vanishes at the special frequencies which have been investigated by Matsuda and Hori $[7,10,11]$. From their results it follows that in the diatomic case $f^{\prime}\left(v^{2}\right)$ has an infinite number of zeros in the interior of the energy band, provided only that the mass-ratio $m_{2} / m_{1}$ is larger than or equal to 1 (without loss of generality let us assume $m_{1} \leqq m_{2}$ ). The analogous result holds for the discrete Schrödinger operator with a random potential $v_{\omega(n)} \in\left\{v_{1}, v_{2}\right\}\left(v_{i} \in \mathbb{R}\right)$, where now the difference $\left|v_{2}-v_{1}\right|$ has to be at least 2 (cf. [22]).

\footnotetext{
${ }^{1}$ Consult references [33-35] for further results concerning the continuity of $k(E)$ in alloy models
} 
To our knowledge the real behaviour of the integrated density of states and of the integrated density of squared frequencies at special energies and special frequencies, respectively, has remained an open problem so far, which was emphasized also by Goda [23]. Moreover, the precise computer calculations by Gubernatis and Taylor [24] for a diatomic Kronig-Penney model show that numerical results have to be interpreted carefully, because their conclusions concerning the behaviour of $k(E)$ near $E_{s}$ are not entirely correct.

In Sect. 2, we present the general method for the determination of the asymptotic behaviour of $k(E)$ at special energies. In Sect. 3, we discuss fixed point configurations of the transfer matrices, which yields sufficient conditions for $\left|k(E)-k\left(E_{s}\right)\right| \sim o\left(\mid E-E_{s}\right)$, i.e. $k^{\prime}\left(E_{s}\right)=0$, and we consider some particular cases with extraordinary asymptotics. The numerical result by Gubernatis and Taylor [24] is discussed in Sect. 4.

The main results (with the exception of Example 3 of Sect. 3) have already been announced in $[25,26]$.

\section{General Method}

Without loss of generality we can assume that the realization $\omega^{\prime}$ of our definition of a special energy denotes the sequence consisting only of 1 . Any other case can be transmitted to this case by transition to a new finite set of transfer matrices, which describes the system, and a corresponding numeration. To simplify our discussion, we will only deal with the Hamiltonian $H^{\omega}$ whose potential $V^{\omega}$ is built up by $V_{1}$ and $V_{2}$ as described in Sect.1,i.e. the case $r=2$. The probability distribution is given by $P\left(V_{i}\right)=c \delta(i-1)+(1-c) \delta(i-2)$ with $c \in(0,1)$. We will treat two typical examples in detail and sketch how to transform the ideas to other cases. Thereby, the key for a uniform treatment of (1), (8) and the discrete Schrödinger operator consists in the possibility of representing the solutions of all these models by transfer matrices (cf., e.g. [27]). If $y$ satisfies $H^{\omega} y=E y$ as a differential equation then we have

$$
\left[\begin{array}{l}
y^{\prime}(n+1) \\
y(n+1)
\end{array}\right]=T_{\omega(n)}\left[\begin{array}{l}
y^{\prime}(n) \\
y(n)
\end{array}\right]
$$

with the random transfer matrix $T_{\omega(n)} \in\left\{T_{1}, T_{2}\right\}$ corresponding to the two types of potentials $V_{1}$ or $V_{2}$, respectively.

For any periodic realization $\omega \in \Omega_{\text {per }}$ it holds that $E \in \sigma\left(H^{\omega}\right)$ if and only if $|\operatorname{Tr} T| \leqq 2$, where $T$ denotes the transfer matrix corresponding to the period of $\omega$ and $\operatorname{Tr} T$ its trace (cf. [28, proof of Theorem XIII.89]). Therefore, the definition of a special energy suggests to distinguish between the following cases $\left(S_{i} \equiv T_{i}\left(E_{s}\right)\right.$, $i=1,2$ ):

Case I: $\quad\left|\operatorname{Tr} S_{1}\right|<2$;

Case II: $\left|\operatorname{Tr} S_{1}\right|=2,\left|\operatorname{Tr} S_{2}\right|>2$,

Case III: $\left|\operatorname{Tr} S_{1}\right|=2,\left|\operatorname{Tr} S_{2}\right|=2$.

Case I has to be further specified. According to the lemma in [8], there exists an integer $n \in N$ such that $S_{1}^{n}=I$. (If such an integer $n$ would not exist, then $S_{1}$ would have eigenvalues $\exp ( \pm \pi i q)$ with $q \in(0,1), q$ irrational. The irrationality of $q$ implies that $\{(n q) \bmod 1 \mid n \in \mathbb{N}\}$ is dense in $[0,1]$, thus $\left\{\operatorname{Tr} S_{1}^{n} S_{2} \mid n \in \mathbb{N}\right\} \cap\left[-\left|\operatorname{Tr} S_{2}\right|\right.$, 


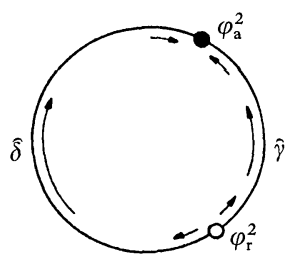

Fig. 1. The fixed point configuration of Example 1. The circular arc represents the projective space, i.e. it is of length $\pi$. The arrows indicate the direction of the $T_{2}$ flow

$\left.\left|\operatorname{Tr} S_{2}\right|\right]$ is dense in $\left[-\left|\operatorname{Tr} S_{2}\right|,\left|\operatorname{Tr} S_{2}\right|\right]$. Hence we could find an integer $n \geqq 1$, such that $\left|\operatorname{Tr} S_{1}^{n} S_{2}\right|<2$. But this contradicts the property (ii) of our definition of a special energy.) Then every random product of matrices can be decomposed into the factors

$$
S_{1}^{n}=I, \quad S_{1} S_{2}, \quad S_{1}^{2} S_{2}, \ldots, S_{1}^{n-1} S_{2}, \quad S_{2},
$$

where $\left|\operatorname{Tr} S_{1}^{k} S_{2}\right| \geqq 2, k=1,2, \ldots, n$.

In the following we will identify the phase $\varphi$ with the corresponding vector of the projective space such that the expression $T_{i} \varphi$ makes sense, where $T_{i} \varphi$ itself also shall be interpreted as an element of the projective space or as the corresponding phase. There is no confusion to fear. The projective space shall be represented by the circular arc $\hat{\pi}$ of length $\pi$ (cf. Fig. 1).

We note that if $S_{1}$ and $S_{2}$ (case II, III) or all matrices $S_{1}^{k} S_{2}, k=1,2, \ldots, n$, (case I) map an $\operatorname{arc} \hat{\delta} \subset \hat{\pi}, \hat{\delta} \neq \hat{\pi}$, into itself, then all products of these matrices as mappings from $\hat{\pi}$ onto $\hat{\pi}$ possess a fixed point. Hence the energy $E_{s}$ under consideration fulfils $E_{s} \notin \operatorname{int} \sigma\left(H^{\omega}\right)$ for any periodic potential $V^{\omega}$ with $\omega \in \Omega_{\text {per }} \backslash\left\{\omega_{1}\right\}$, and thus it satisfies the definition of a special energy.

Example 1. Assume that we have case II with $S_{1}=I$. Of course typically $\left|\operatorname{Tr} S_{1}\right|=2$ does not mean $S_{1}= \pm I$. $S_{1}=I$ occurs when there are two periodic solutions to the particular energy. (Then $\operatorname{Tr} T_{1}(E)$ achieves a maximum at $E_{s}$.) Then (cf. [28, proof of Theorem XIII.89]) for every sufficiently small $\varepsilon>0$ it holds that

$$
T_{1}\left(E_{s} \pm \varepsilon\right)=X_{ \pm} D_{\psi_{ \pm}} X_{ \pm}^{-1},
$$

where $D_{\psi}$ denotes a rotation matrix turning every vector by the angle $\psi$ and $X$ is some regular matrix. The asymptotic behavior of $\psi$ is given by $\psi_{ \pm}(\varepsilon) \sim \pm \alpha \varepsilon$ for $\varepsilon \rightarrow 0$ (cf. [29, Sect. 4]), where the positive constant $\alpha$ can be obtained from the Taylor expansion of $\operatorname{Tr} T_{1}$, cf. Sect. 4. Let $\varphi_{a}^{2}$ be the attractive fixed point of $S_{2}$ and $\varphi_{r}^{2}$ the repulsive one. Further, we denote by $\hat{\delta}$ the $\operatorname{arc}\left[\varphi_{a}^{2}, \varphi_{r}^{2}\right]\left(\delta \equiv \varphi_{r}^{2}-\varphi_{a}^{2}\right)$ and by $\hat{\gamma}$ the $\operatorname{arc}\left[\varphi_{r}^{2}, \varphi_{a}^{2}\right]\left(\gamma \equiv \varphi_{a}^{2}-\varphi_{r}^{2}=\pi-\delta\right)$, cf. Fig. 1 .

For $E=E_{s}$ all products of $S_{1}$ and $S_{2}$ map $\hat{\delta}$ and $\hat{\gamma}$ into itself. When the energy increases by $\varepsilon, \varepsilon \ll 1$, then phase rotations are possible in consequence of the action of the matrices $T_{1}$. Thereby the critical region which has to be conquered is $\left[\varphi_{a}^{2}+\varepsilon^{p}, \varphi_{r}^{2}-\varepsilon^{p}\right], p<1$, since here $T_{2}$ rotates every phase at least of order $\varepsilon^{p}$ in negative direction like a simple calculation shows. Namely, one finds that for $\varepsilon \rightarrow 0$ $T_{2}\left(\varphi_{a}^{2}+\varepsilon\right)$ and $T_{2}\left(\varphi_{r}^{2}-\varepsilon\right)$ behaves as $\varphi_{a}^{2}+C_{1} \varepsilon$ and $\varphi_{r}^{2}-C_{2} \varepsilon$, respectively, where $C_{1}>1$ and $C_{2}>1$ are some constants. Now, using relation (4) we refine the ideas of 
[6] in the following way: Let $\hat{\delta}^{\prime} \equiv\left[X_{+}^{-1}\left(\varphi_{a}^{2}+\varepsilon^{p}\right), X_{+}^{-1}\left(\varphi_{r}^{2}-\varepsilon^{p}\right)\right]$. Then for every realization $\omega$, it obviously holds that

$$
\frac{\varphi_{E_{s}+\varepsilon}^{\omega}(L)-\varphi_{E_{s}}^{\omega}(L)}{\pi} \leqq R_{L}^{\omega}+\Omega_{L}^{\omega}
$$

where $R_{L}^{\omega}$ denotes the number of disjoint parts of length $\left[\delta^{\prime} /(\alpha \varepsilon)\right]$ between 0 and $L$ which are composed only of matrices $T_{1}$, and $\Omega_{L}^{\omega}$ is the number of phase runnings through $\hat{\delta}^{\prime}$ which are due to mixed parts of the chain. [x] denotes the integral part of $x . \Omega_{L}^{\omega}$ can be neglected since the probability of such an event tends to zero in a higher order than that of the event corresponding to $R_{L}^{\omega}$. [Here we used that in $\hat{\delta}^{\prime} T_{2}$ rotates every phase at least of order $\varepsilon^{p}$ whereas at least $i \varepsilon^{p} /(\alpha \varepsilon)$ matrices $T_{1}$ are necessary to compensate the rotation effect of $i$ matrices $T_{2}$ in $\hat{\delta}^{\prime}$.]

Taking the limit $L \rightarrow \infty$, we obtain by the law of large numbers

$$
k\left(E_{s}+\varepsilon\right)-k\left(E_{s}\right) \leqq c^{\delta^{\prime} /(\alpha \varepsilon)}+o\left(c^{\delta^{\prime} /(\alpha \varepsilon)}\right)
$$

or

$$
\lim _{\varepsilon \rightarrow 0} \varepsilon \ln \left(k\left(E_{s}+\varepsilon\right)-k\left(E_{s}\right)\right) \leqq \frac{\delta^{*} \ln c}{\alpha}
$$

with $\delta^{*}=X_{+}^{-1} \varphi_{r}^{2}-X_{+}^{-1} \varphi_{a}^{2}$.

A lower bound is obtainable by counting all pure $T_{1}$-parts of minimal length $\delta^{\prime \prime} /(\alpha \varepsilon) \equiv\left(X_{+}^{-1}\left(\varphi_{r}^{2}+\varepsilon^{p}\right)-X_{+}^{-1}\left(\varphi_{a}^{2}-\varepsilon^{p}\right)\right) /(\alpha \varepsilon)$ which are directly preceding at least $\left[\left(\gamma-2 \varepsilon^{p}\right) / \varepsilon^{p}\right]$ matrices of type $T_{2}$. Thereby the number of matrices $T_{2}$ guarantees that the $\operatorname{arc} \delta$ will be really conquered by every of these parts. In analogy to above we obtain

$$
k\left(E_{s}+\varepsilon\right)-k\left(E_{s}\right) \geqq(1-c)^{\left(\gamma-2 \varepsilon^{p}\right) / \varepsilon^{p}} c^{\delta^{\prime \prime} /(\alpha \varepsilon)}+o\left(c^{\delta^{\prime \prime} /(\alpha \varepsilon)}\right)
$$

or

$$
\lim _{\varepsilon \rightarrow 0} \varepsilon \ln \left(k\left(E_{s}+\varepsilon\right)-k\left(E_{s}\right)\right) \geqq \frac{\delta^{*} \ln c}{\alpha} .
$$

The expression $\left|k\left(E_{s}+\varepsilon\right)-k\left(E_{s}\right)\right| \sim \exp \left(\delta^{*} \ln c /(\alpha \varepsilon)\right)$ shall be understood as an abbreviation of (10) and (11). The considerations are analogous for the case $E_{s}-\varepsilon$. Only $\delta^{*}$ has to be replaced by $\gamma^{*} \equiv X_{-}^{-1} \varphi_{a}^{2}-X_{-}^{-1} \varphi_{r}^{2}$.

Example 2. Now, let us consider the more complicated case I with $S_{1}^{2}=I$ and $\left|\operatorname{Tr} S_{2}\right|>2,\left|\operatorname{Tr} S_{1} S_{2}\right|=2$. Of course, case I is in general more typical than the cases II and III, since the condition $\left|\operatorname{Tr} S_{1}\right|=2$ is more restrictive than $\left|\operatorname{Tr} S_{1}\right|<2$. By the same reason $\left|\operatorname{Tr} S_{1} S_{2}\right|>2$ is for the Hamiltonian (1) and (2) more typical than the condition $\left|\operatorname{Tr} S_{1} S_{2}\right|=2$. But $\left|\operatorname{Tr} S_{1} S_{2}\right|>2$ at most yields the asymptotics (10) and (11) as the following consideration shows. Thus, our example is very illustrative, and it had been observed in various models discussed earlier. We will assume the fixed point configuration $\varphi_{\mathrm{a}}^{2} \rightarrow \varphi_{\mathrm{ar}}^{12} \rightarrow \varphi_{\mathrm{r}}^{2}$, where $\varphi_{\mathrm{ar}}^{12}$ is the fixed point of $T_{1} T_{2}$ and the order of the indices, namely ar, denotes the order of the attractive and repulsive side of this fixed point going through it in a mathematically positive sense, cf. Fig. 2. 

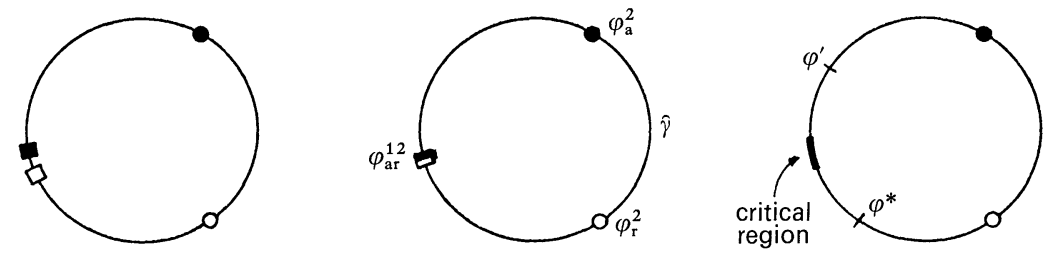

Fig. 2. The fixed point configuration of Example 2. For $E=E_{s}-\varepsilon$ the fixed point $\varphi_{\text {ar }}^{22}$ splits up into two fixed points, whereas for $E=E_{s}+\varepsilon$ the product $T_{1} T_{2}$ has no fixed point

Every finite random product of these transfer matrices can be decomposed into the factors $S_{1}^{2}, S_{1} S_{2}, S_{2}$, which map $\left[\varphi_{\mathrm{a}}^{2}, \varphi_{\mathrm{ar}}^{12}\right]$ into itself. When the energy decreases by $\varepsilon$ the considerations are the same as in Example 1 since $\hat{\gamma}$ again is the critical region, which can only be overcome by the factors $T_{1}^{2}\left(E_{s}-\varepsilon\right)$, now. Thus, the asymptotic behaviour is determined by

$$
k\left(E_{s}\right)-k\left(E_{s}-\varepsilon\right) \sim \exp \left(2 \gamma^{*} \ln c /(\alpha \varepsilon)\right)
$$

with some positive constant $\alpha$ depending on $T_{1}^{2}$ as stated in Example 1 .

In the case $E_{\mathrm{s}}+\varepsilon, \varepsilon$ small and positive, we have not only $T_{1}^{2}\left(E_{s}+\varepsilon\right)=X D_{\psi} X^{-1}$, but also $T_{1} T_{2}\left(E_{s}+\varepsilon\right)=Y D_{\theta} Y^{-1}$, since $\left|\operatorname{Tr} T_{1} T_{2}\left(E_{s}+\varepsilon\right)\right|<2$, what changes our consideration in comparison with $E=E_{\mathrm{s}}-\varepsilon$. Whereas $\psi \sim \alpha \varepsilon, \theta$ behaves as $\beta \varepsilon^{1 / 2}$ for $\varepsilon \rightarrow 0, \beta$ is a positive constant (cf. [30, Lemma B1]). Thus, in first order only the matrices $T_{1} T_{2}$ (and $T_{2}$ in $\left[\varphi_{\mathrm{r}}^{2}, \varphi_{\mathrm{a}}^{2}\right]$ ) contribute to a phase rotation. To overcome the critical region near the fixed point $\varphi_{\mathrm{ar}}^{12}\left[\pi /\left(\beta \varepsilon^{1 / 2}\right)\right]$ factors $T_{1} T_{2}$ are needed up to some constant, since every closed $\operatorname{arc} \delta$ with $\varphi_{\text {ar }}^{12} \notin \hat{\delta}$ can be conquered by a finite number of matrices $T_{1} T_{2}$. Nevertheless, we cannot neglect the matrices $T_{1}^{2}$ since the probability of any finite product of factors $T_{1} T_{2}$ accomplished by matrices $T_{1}^{2}$ is of the same order as those of the pure product. Compared to it, parts in which matrices $T_{2}$ bring the phase back beyond $\varphi_{\mathrm{ar}}^{12}$ obviously have probability of higher order. If the matrix $T_{2}$ does not rotate the phase $\varphi \in\left[\varphi_{\mathrm{a}}^{2}, \varphi_{\mathrm{r}}^{2}\right]$ back beyond $\varphi_{\mathrm{ar}}^{12}$, then in each case at most some finite number (independent of $\varepsilon$ ) of matrices $T_{1} T_{2}$ is necessary to compensate the rotation in the negative direction. But this only influences the factor $A$, see (14). For a lower estimate we have to count all parts of $\left[\pi /\left(2 \beta \varepsilon^{1 / 2}\right)\right]-m$ matrices $T_{1} T_{2}, m<\infty$, possibly accomplished by matrices $T_{1}^{2}$ (they rotate the phase over $\left[\varphi_{\mathrm{ar}}^{12}, \varphi^{*}\right]$, where $\left.T_{2} \varphi^{*}=\varphi_{\mathrm{ar}}^{12}\right)$, followed by a mixed part of matrices $T_{1}^{2}, T_{1} T_{2}, T_{2}$ which guarantees that the phase conqueres the $\operatorname{arc}\left(\varphi^{*}, \varphi_{\mathrm{ar}}^{12}\right]$. Thereby again $\left[\pi /\left(2 \beta \varepsilon^{1 / 2}\right)\right]-m^{\prime}$ matrices $T_{1} T_{2}, m^{\prime}<\infty$, accomplished by matrices $T_{1}^{2}$ are needed to reach $\varphi_{\mathrm{ar}}^{12}$, i.e. to conquer $\left[\varphi^{\prime}, \varphi_{\mathrm{ar}}^{12}\right]$ with $T_{2} \varphi_{\mathrm{ar}}^{12}=\varphi^{\prime}$. The probability $P_{0}$ of a mixed chain of $n \equiv\left[\pi /\left(\beta \varepsilon^{1 / 2}\right)\right]$ matrices $T_{1} T_{2}$ and matrices $T_{1}^{2}$ is given by

$$
P_{0}=(c(1-c))^{n} \sum_{i=0}^{\infty}\left(\begin{array}{c}
n+i-2 \\
i
\end{array}\right) c^{2 i}=\left(\frac{c}{1+c}\right)^{n}\left(1-c^{2}\right),
$$

where we used $\sum_{i=0}^{\infty}\left(\begin{array}{c}m+1 \\ i\end{array}\right) d^{i}=\left(1+d+d^{2}+d^{3}+\ldots\right)^{m+1}$. [Equation (13) ensures that there is a factor $T_{1} T_{2}$ at the beginning and at the end of the chain, which 
excludes double countings.] Thus, we get

$$
k\left(E_{s}+\varepsilon\right)-k\left(E_{s}\right) \leqq A \exp \left(\pi \ln \frac{c}{1+c} /\left(\beta \varepsilon^{1 / 2}\right)\right)
$$

with some constant $A<\infty$. Otherwise, counting only parts of $\left.\left[\pi / \beta \varepsilon^{1 / 2}\right)\right]$ matrices $T_{1} T_{2}$ we obviously get

$$
k\left(E_{s}+\varepsilon\right)-k\left(E_{s}\right) \geqq \exp \left(\pi \ln \frac{c}{1+c} /\left(\beta \varepsilon^{1 / 2}\right)\right) .
$$

In comparison with Example 1, in Example 2 we obtain a stronger estimate since we have no regions depending on $\varepsilon$, in which the rotations of different matrices compensate each other.

With these two examples the principal technique of handling the typical cases of special energies and special frequencies, respectively, has been given. We see that the asymptotic behaviour of $k(E)$ depends on the fixed point configuration. It is even possible for all cases (I, II, III) to find such configurations that $k^{\prime}\left(E_{s}\right) \neq 0$. In the next section, we will deal with this problem and with some examples of various asymptotics.

\section{Fixed Point Configurations}

Now it is easy to verify that there are the following five types of asymptotics or combinations of the behaviour of $k(E)$ to the left and right of a special energy $E_{s}$ $\left(\varepsilon \equiv\left|E-E_{s}\right|\right)$ :

Type 1: $\exp (-A / \varepsilon), \exp (-B / \varepsilon)$

Type 2: $\exp (-A / \varepsilon), \exp \left(-B / \varepsilon^{1 / 2}\right)$,

Type 3: $\exp \left(-A / \varepsilon^{1 / 2}\right), \exp \left(-B / \varepsilon^{1 / 2}\right)$,

Type 4: $\varepsilon^{1 / 2}, \exp (-B / \varepsilon)$,

Type 5: $\varepsilon, \varepsilon$.

Thereby it is trivial that there is no favourable side for any of the asymptotics because you only have to reflect the fixed point configuration to change the sides. The first and the second possibility of (16) correspond to Examples 1 and 2, respectively. In the preceding section, we already explained that Type 1 has to be considered as the typical asymptotic of $k(E)$ at special energies of the Hamiltonian (1) and (2). In the case of the discrete Schrödinger operator or the Hamiltonian (8) the very special form of the transfer matrices causes that in case I always $\left|\operatorname{Tr} S_{1}^{n-1} S_{2}\right|=2$, where $S_{1}^{n}=I$. Thus, for these Hamiltonians Type 2 is typical (cf. Example 2). Assuming, e.g., in case III the fixed point configuration $\varphi_{\mathrm{ra}}^{1} \rightarrow \varphi_{\mathrm{ar}}^{2}$, one gets the third combination. The considerations of Example 1 are easily applicable to case III with $S_{1}=I$ and $S_{2}$ having a fixed point $\varphi_{\text {ra }}^{2}$, and they yield type 4 . In the same case, assuming $S_{1}=I$ and $S_{2}=I$, we get the last asymptotic.

The possibility that $E_{s}$ is a band edge has not been considered here since it is not of interest for us. However, type 3 may be regarded as two band edges put together, 
whereas types $1,2,4$, and 5 occur within the band. The asymptotic $\varepsilon^{1 / 2}$ is typical for a "stable" band edge, cf. [6].

We note that $k^{\prime}\left(E_{s}\right)$ has not to vanish or even has not to exist. Type 4 exhibits $k^{\prime}\left(E_{s}-0\right)=\infty, k^{\prime}\left(E_{s}+0\right)=0$. Type 5 shows $k^{\prime}\left(E_{s}\right)>0$.

It seems to us that it is impossible to give a complete distinction of all cases of special energies. Let us consider the case I, the most important case since the other ones are exceptional cases, and let us assume that at most two of the matrices $S_{1}^{k} S_{2}$, $k=1, \ldots, n\left(S_{1}^{n}=I\right)$, satisfy $\left|\operatorname{Tr} S_{1}^{k} S_{2}\right|=2$ with $S_{1}^{k} S_{2} \neq I$. Then we have the following

Proposition. Assume that the fixed point configuration of the matrices $S_{1}^{k} S_{2}$, $k=1,2, \ldots, n$, admits a decomposition of $\hat{\pi}$ into four successive arcs $\hat{\delta}_{\mathrm{a}} \rightarrow \widehat{\delta}_{1} \rightarrow \widehat{\delta}_{\mathrm{r}} \rightarrow \hat{\delta}_{2}$, where $\widehat{\delta}_{\mathrm{a}}$ contains all attractive fixed points $\varphi_{\mathrm{a}}$ (there shall be at least one) and $\hat{\delta}_{\mathrm{r}}$ all repulsive ones; $\widehat{\delta}_{\mathrm{a}} \cap \widehat{\delta}_{\mathrm{r}}=\emptyset$. If there are no other fixed points, then the asymptotic behaviour is of type 1. If there is additionally exactly one fixed point $\varphi_{\mathrm{ar}} \in \widehat{\delta}_{1}$ or $\varphi_{\mathrm{ra}} \in \widehat{\delta}_{2}$ or if both of them occur, then the asymptotic behaviour is of type 2 or 3, respectively.

The proof just follows the lines of the Examples 1 and 2, where $\widehat{\delta}_{1}$ and $\widehat{\delta}_{2}$ play the role of $\hat{\delta}$ and $\hat{\gamma}$, respectively. Likewise, the proposition immediately extends to the case of $r$ transfer matrices, $r>2$.

The very special fixed point configuration $\varphi_{\mathrm{r}}^{1}=\varphi_{\mathrm{a}}^{2} \rightarrow \varphi_{\mathrm{a}}^{1}=\varphi_{\mathrm{r}}^{2}$ (Fig. 3), where we will suppose $S_{1}^{-1}=S_{2}$, yields a further type of asymptotics. Now $\omega^{\prime}$ in our definition of a special energy is equal to the sequence $(\ldots, 1,2,1,2,1,2, \ldots)$.

The investigation of the special energy corresponding to Fig. 3 requires a modification of the method presented in our previous examples, which leads us to

Example 3. Let us consider the case $E=E_{s}+\varepsilon, \varepsilon>0$. Whereas for $\varepsilon=0, S_{1}$, and $S_{2}$ map $\left[\varphi_{\mathrm{a}}^{1}, \varphi_{\mathrm{r}}^{1}\right]$ and $\left[\varphi_{\mathrm{r}}^{1}, \varphi_{\mathrm{a}}^{1}\right]$ into itself, now phase rotations are possible since $T_{1} T_{2}\left(E_{s}+\varepsilon\right)=X D_{\psi} X^{-1}$ with $\psi(\varepsilon) \sim \alpha \varepsilon$ for $\varepsilon \rightarrow 0$. Furthermore, the transfer matrix $T_{1}\left(E_{s}+\varepsilon\right)$ maps the phase $\varphi_{\mathrm{r}}^{T_{1}}+x \varepsilon, x \ll 1$, to $\varphi_{\mathrm{r}}^{T_{1}}+\beta x \varepsilon$ with $\beta>1$, as one can easily check by an explicit calculation. In the region $\left[\varphi_{\mathrm{r}}^{T_{1}}+\varepsilon^{p},\left(\varphi_{\mathrm{r}}^{T_{1}}+\varphi_{\mathrm{a}}^{T_{1}}\right) / 2\right]$ shown in Fig. 3, $p<1, T_{1}$ rotates over an angle of higher order than $T_{1} T_{2}$ does. Therefore, $n_{1}=[-p \ln \varepsilon / \ln \beta]$ matrices $T_{1}$ are necessary up to some constant to overcome this region. The situation is analogous in $\left[\left(\varphi_{\mathrm{r}}^{T_{1}}+\varphi_{\mathrm{a}}^{T_{1}}\right) / 2, \varphi_{\mathrm{a}}^{T_{1}}-\varepsilon^{p}\right]$, where $n_{2}=\left[-p \ln \varepsilon / \ln \beta^{\prime}\right]$ matrices $T_{1}$ are needed with some $\beta^{\prime}>1$.

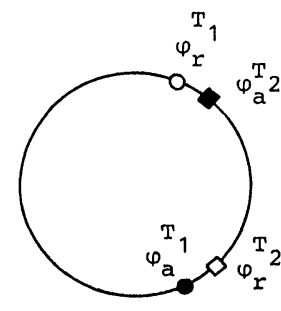

$E=E_{S}-\varepsilon$

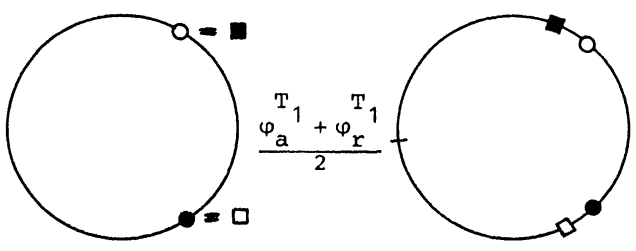

$E=E_{S}$
$E=E_{S}+\varepsilon$

Fig. 3. The fixed point configuration of Example 3 
The further investigation depends on the probability $c$ with which the matrix $T_{1}$ occurs. First, let $c<1 / 2$ and $n=n_{1}+n_{2}=-p A \ln \varepsilon$ with $A=\ln \left(\beta \beta^{\prime}\right) / \ln \beta \ln \beta^{\prime}$. We denote by $K(n, x)$ a finite chain consisting of $n(1+x)$ matrices $T_{1}$ and $n x$ matrices $T_{2}$. The probability of such a chain is given by $W(n, x)=\left(\begin{array}{c}n+2 n x \\ n x\end{array}\right) c^{n+n x}(1-c)^{n x}$. Thus, we get

$$
\max _{x} W(n, x)=W\left(n, x_{m}\right) \approx\left(\frac{c}{1-c}\right)^{n}, \quad x_{m}=\frac{c}{1-2 c}
$$

by Stirling's formula.

We obtain an upper bound for the integrated density of states decomposing the infinite random chain of transfer matrices into parts of length $(c /(1-c)+\delta)^{-n}$ with $\delta>0$. With probability 1 there is for $\varepsilon \rightarrow 0$ no chain $K(n, x), x$ arbitrary, in such a part, i.e. there is no phase rotation in it: Obviously, the probability of chains $K(n, x)$ with $x<n$ and arbitrary starting point in such a part is smaller than $(c /(1-c)+\delta)^{-n}(c /(1-c))^{n} n^{2}$, tending to zero with $n \rightarrow \infty$. The probability of $K(n, x)$ with $k n \leqq x<(k+1) n, k \in N$, in such a part is smaller than $n^{2} 2^{n}(4 c(1-c))^{k n^{2}}$ by Stirling's formula. Of course, the sum over $k$ (geometrical series) tends to zero for $n \rightarrow \infty$. Thus, we have

$$
\begin{aligned}
k\left(E_{s}+\varepsilon\right)-k\left(E_{s}\right) \leqq & \varepsilon^{-p A \ln (c /(1-c)+\delta)} \\
& +o\left(\varepsilon^{-p A \ln (c /(1-c)+\delta)}\right)
\end{aligned}
$$

with arbitrary positive constants $p<1$ and $\delta$.

Now, let $n=-A \ln \varepsilon$, i.e. we choose $p=1$. Then a chain $K\left(n, x_{m}\right)$ and an analogous chain $K^{*}$ in which the portions of $T_{1}$ and $T_{2}$ are just changed yield a phase rotation if we only add some constant number of matrices to conquer $\left[\varphi_{\mathrm{a}}^{1}-\varepsilon, \varphi_{\mathrm{r}}^{2}+\varepsilon\right]$ and $\left[\varphi_{\mathrm{a}}^{2}-\varepsilon, \varphi_{\mathrm{r}}^{1}+\varepsilon\right]$. Thereby the probability of $K^{*}$ is of higher order than (17). Thus, if we decompose the chain of transfer matrices into parts of length $(c /(1-c)-\delta)^{-n}$ and every part in smaller sub-chains of length $n\left(1+x_{m}\right)$, then we obtain a lower bound for $k(E)$ using that

$$
\left(1-\left(\begin{array}{c}
c \\
1-c
\end{array}\right)^{n}\right)^{1 /\left((c(1-c)-\delta)^{n} n\left(1+x_{m}\right)\right)} \rightarrow 0
$$

for $n \rightarrow \infty$, i.e. for $\varepsilon \rightarrow 0$ we have with probability 1 at least one phase rotation in each of these parts. Therefore, for $\varepsilon \rightarrow 0$ it follows that

$$
k\left(E_{s}+\varepsilon\right)-k\left(E_{s}\right) \geqq \varepsilon^{-A \ln (c /(1-c)-\delta)}+o\left(\varepsilon^{-A \ln (c /(1-c)-\delta)}\right),
$$

where $\delta$ is an arbitrary small positive constant.

If $c>1 / 2$ then the regions $\left[\varphi_{\mathrm{a}}^{1}, \varphi_{\mathrm{r}}^{1}\right]$ and $\left[\varphi_{\mathrm{r}}^{1}, \varphi_{\mathrm{a}}^{1}\right]$ only change their roles and we get the same result.

In the case $c=1 / 2$ we consider parts of length $\left[|\ln \varepsilon|^{2}\right]$. The probability that such a part contains at least $[|A \ln \varepsilon|]$ matrices of one type more than of the other type is larger than some constant $q>0$ independent of $\varepsilon$ (cf. [31, Sect. 7.3]). Since two of these parts with exchanged favourable matrices guarantee a phase rotation, for $\varepsilon \rightarrow 0$ we get by the law of large numbers

$$
k\left(E_{\mathrm{s}}+\varepsilon\right)-k\left(E_{\mathrm{s}}\right) \geqq B|\ln \varepsilon|^{-2}
$$


with some constant $B>0$. Thus $k^{\prime}\left(E_{s}+0\right)=\infty$ for $c=1 / 2$. On the other hand, it obviously holds that

$$
k\left(E_{s}+\varepsilon\right)-k\left(E_{s}\right) \leqq|\ln \varepsilon|^{-1} .
$$

For $E=E_{s}-\varepsilon$ the considerations are analogous by reason of the symmetry of the fixed point configuration.

\section{Comparison with Computer Experiments}

In this section, we ascertain the exact asymptotic behaviour of the integrated density of states at a special energy investigated by computer calculations, and compare it with the numerical result.

Gubernatis and Taylor [24] deal with the Hamiltonian (1), (2), where $V_{\omega(n)}=v_{\omega(n)} \delta(x-n)$ with $v_{1}=-0.4$ and $v_{2}=-4$, i.e. they consider a random Kronig-Penney model. (The phase $\varphi(x)$ is discontinuous in $n \in Z$, but unambiguously defined if we postulate $\varphi(n-0)$ and $\varphi(n+0)$ to be in the same branch of the arc tangent.) From computer calculations, Gubernatis and Taylor conclude the asymptotic behaviour

$$
\left|k\left(E_{s} \pm \varepsilon\right)-k\left(E_{s}\right)\right| \sim \exp \left(-c_{ \pm} / \varepsilon^{1 / 2}\right)
$$

at special energies. The energy $E_{s}$ considered by them is implicitly given by $\tan k_{s}=5 k_{s}$, where $k=E^{1 / 2}$ (cf. [32]). Assuming that the strength of the $\delta$-potential is equally distributed, i.e. $c=1 / 2$, they calculate $c_{-}=5.566$ and $c_{+}=1.402$.

In this model, the transfer matrices are of the form

$$
T_{i}(k)=\left[\begin{array}{cc}
\cos k & -k \sin k+v_{i} \cos k \\
\frac{\sin k}{k} & \cos k+v_{i} \frac{\sin k}{k}
\end{array}\right]
$$

(cf. [32]). One easily proves $\operatorname{Tr} S_{1}=0, S_{1}^{2}=I,\left|\operatorname{Tr} S_{2}\right|>2$, and $\left|\operatorname{Tr} S_{1} S_{2}\right|=2$. Further, the fixed point configuration is the same as shown in Fig. 2, so that we are just in the case of Example 2. Instead of (22) we obtain (12) for the behaviour of the integrated density of states on the left of $E_{s}$. To calculate $\alpha$ we have to regard $T_{1}^{2}\left(E_{s}-\varepsilon\right)$ for $\varepsilon \rightarrow 0$. We abbreviate $\tau \equiv\left|k-k_{s}\right| \sim \varepsilon / 2 k_{s}$. Using the equation for $k_{s}$ we obtain in terms up to second order in $\tau$

$$
T_{1}^{2}\left(k_{s}-\tau\right) \sim\left[\begin{array}{cc}
-1+\tau\left(10 k_{s}-1.6 / k_{s}\right) w & \tau\left(-50 k_{s}^{3}+4 k_{s}+0.64 / k_{s}\right) w \\
+\tau^{2}\left(50 k_{s}^{2}-8-1.6 / k_{s}^{2}\right) w & +\tau^{2}\left(40 k_{s}^{2}+1.6+0.64 / k_{s}^{2}\right) w \\
\tau\left(50 k_{s}-8 / k_{s}\right) w & -1+\tau\left(-10 k_{s}+1.6 / k_{s}\right) w \\
+\tau^{2}\left(40-14.4 / k_{s}^{2}\right) w & +\tau^{2}\left(50 k_{s}^{2}+4.16 / k_{s}^{2}-24\right) w
\end{array}\right],
$$

where $\quad w=\cos ^{2} k_{s}$ Therefore, we get with $k_{s} \approx 1.432032\left|\operatorname{Tr} T_{1}^{2}\left(k_{s}-\tau\right)\right|$ $\sim 2-3.3351 \tau^{2}$. Since $\operatorname{Tr} T_{1}^{2}\left(E_{s}-\varepsilon\right)=\exp \left(i \psi_{-}\right)+\exp \left(-i \psi_{-}\right)=2 \cos \psi_{-}$, we have $\psi_{-}(\varepsilon) \sim-0.6376 \varepsilon$, i.e. $\alpha \approx 0.6376$. To calculate $\gamma^{*}$ we have to compute $X_{-}^{-1}$ and apply it to the fixed points $\varphi_{\mathrm{a}}^{2} \approx(1,1.0776)^{T}, \varphi_{\mathrm{r}}^{2} \approx(1,0.3255)^{T}$. With

$$
X_{-}^{-1} \approx\left[\begin{array}{cc}
1 & 0 \\
-0.1383 & 1.4736
\end{array}\right] \text {, }
$$


it follows that $\gamma^{*} \approx 0.638$ and

$$
k\left(E_{s}\right)-k\left(E_{s}-\varepsilon\right) \sim \exp (-1.387 / \varepsilon), \quad \varepsilon \rightarrow+0 .
$$

The asymptotic behaviour on the other side of $E_{s}$ is of type (14),(15). To calculate $\beta$ we have to expand $T_{1} T_{2}\left(k_{s}+\varepsilon\right)$ :

$$
T_{1} T_{2}\left(k_{s}+\tau\right) \sim\left[\begin{array}{cc}
-1+\tau\left(-10 k_{s}+1.6 / k_{s}\right) w & 3.6+\tau\left(50 k_{s}^{3}+32 k_{s}-6.4 / k_{s}\right) w \\
\tau\left(-50 k_{s}+8 / k_{s}\right) w & -1+\tau\left(190 k_{s}-30.4 / k_{s}\right) w
\end{array}\right] .
$$

Thus we have $\left|\operatorname{Tr} T_{1} T_{2}\left(k_{s}+\tau\right)\right| \sim 2-4.5469 \tau$, and therefore $\beta \approx 1.25998$. We obtain

$$
k\left(E_{s}+\varepsilon\right)-k\left(E_{s}\right) \sim \exp \left(-2.739 / \varepsilon^{1 / 2}\right), \quad \varepsilon \rightarrow+0 .
$$

The comparison with (22) reveals in both cases the limited value of the numerical results obtained by Gubernatis and Taylor for the behaviour of $k(E)$ at special energies.

Acknowledgements. It is a pleasure to thank L. A. Pastur and T. Spencer for helpful comments. One of the authors (H.E.) thanks S. Albeverio, Ph. Blanchard, and L. Streit for the invitation to Bielefeld, where this revised version was finished, and Mrs. L. Jegerlehner for her nice typing.

\section{References}

1. Reed, M., Simon, B.: Methods of modern mathematical physics. II. New York: Academic Press 1975

2. Englisch, H.: One-dimensional Schrödinger operators with ergodic potential. Z. Anal. Anw. 2, 411-426 (1983)

3. Pastur, L.A.: Spectra of random self-adjoint operators. Usp. Mat. Nauk. 28, 3-64 (1973)

4. Kirsch, W., Martinelli, F.: On the density of states of Schrödinger operators with a random potential. J. Phys. A 15, 2139-2156 (1982)

5. Englisch, H.: The phase formalism for one-dimensional Schrödinger operators with a random potential. Preprint ICTP Trieste, to appear in Rept. Math. Phys.

6. Gredescul, S.A., Pastur, L.A.: Behaviour of the density of states in one-dimensional disordered systems near the spectrum bounds. Teor. Mat. Fiz. 23, 132-139 (1975)

7. Matsuda, H.: Special frequencies in the vibrational spectra of disordered chains. Prog. Theor. Phys. 31, 161-162 (1964)

8. Englisch, H.: Discontinuities in disordered systems. Z. Phys. B 53, 289-293 (1983)

9. Dean, P.: Vibrational spectra of diatomic chains. Proc. R. Soc. Lond. A 254, 507 (1960)

10. Hori, J., Matsuda, H.: Structure of the spectra of disordered systems. Prog. Theor. Phys. 32, 183-189 (1964)

11. Hori, J.: Phase theory of disordered systems. Supp. Prog. Theor. Phys. 36, 3-54 (1966)

12. Englisch, H., Kürsten, K.-D.: Infinite-representability of Schrödinger operators with ergodic potential. Z. Anal. Anw. 3, 357-366 (1984)

13. Kirsch, W., Martinelli, F.: On the spectrum of Schrödinger operators with a random potential. Commun. Math. Phys. 85, 329-350 (1982)

14. Avron, J., Simon, B.: Almost periodic Schrödinger operators. II. The integrated density of states. Duke Math. J. 50, 369-391 (1983)

15. Craig, W., Simon, B.: Log Hölder continuity of the integrated density of states for stochastic Jacobi matrices. Commun. Math. Phys. 90, 207-218 (1983)

16. Nieuwenhuizen, T.M.: Exact electronic spectra and inverse localization lengths in onedimensional random systems. Physica 120 A, 468-514 (1983) 
17. Lifšic, I.M.: Energy spectrum structure and quantum states of disordered condensed systems. Sov. Phys. Usp. 7, 549 (1965)

[For a modern review cf. Simon, B.: Lifschitz tails for the Anderson model. J. Stat. Phys. 38, 65-76 (1985)]

18. Simon, B.: Fifteen problems in mathematical physics. In: Perspectives of mathematics. Basel: Birkhäuser, pp. 423-454

19. Wegner, F.: Bounds on the density of states in disordered systems. Z. Phys. B 44, 9-15 (1981)

20. Lieb, E.H., Mattis, D.C.: Mathematical physics in one dimension. New York: Academic Press 1966

21. Miyazima, S.: On the special frequency in a vibrational linear chain with random masses and force-constants. Prog. Theor. Phys. 53, 929-935 (1975)

22. Gubernatis, J.E., Taylor, P.L.: Some details of the density of states of a disordered alloy. J. Phys. C6, 1889-1895 (1973)

23. Goda, M.: In: Physics in one dimension. Bernasconi, J., Schneider, T. (eds.). Berlin, Heidelberg, New York: Springer 1981

24. Gubernatis, J.E., Taylor, P.L.: Special aspects of the electronic structure of a one-dimensional random alloy. J. Phys. C 4, L94-L96 (1971)

25. Englisch, H.: Random alloys and special energies. Preprint, MECO-Seminar 1984, Gernrode, GDR

26. Englisch, H., Endrullis, M.: Random alloys and special energies. Abstracts of Colloquium and workshop on random fields, Köszeg, Hungary 1984

27. Endrullis, M., Englisch, H.: Bounds on the Ljapunov exponent. Ann. Phys. (Leipzig) 41, 303-314 (1984)

28. Reed, M., Simon, B.: Methods of modern mathematical physics. IV. New York: Academic Press 1978

29. Moser, J.: An example of a Schrödinger equation with almost periodic potential and nowhere dense spectrum. Comment. Math. Helv. 56, 198-224 (1981)

30. Avron, J., Simon, B.: Analytic properties of band functions. Ann. Phys. (New York) 110, 85-101 (1978)

31. Feller, W.: An introduction to probability theory and its application, Vol. I. New York: Wiley 1970

32. Wada, K.: Special energies in the electronic energy spectrum for a disordered diatomic chain. Prog. Theor. Phys. 36, 726-733 (1966)

33. Simon, B., Taylor, M.: Harmonic Analysis on $S L(2, R)$ and smoothness of the density of states in the one-dimensional Anderson model. Commun. Math. Phys. 101, 1-19 (1985)

34. Nieuwenhuizen, T.M., Luck, J.M.: Singular behaviour of the density of states and the Lyapunov coefficient in binary random harmonic chains. J. Stat. Phys. 41, 745 (1985)

35. Martinelli, F.: Talk at the Oberwolfach meeting "Mathematical Problems in Condensed Matter Physics", 31.3.-4.4.1986

Communicated by T. Spencer

Received June 3, 1985; in revised form August 18, 1986

Note added in proof. Similar results are derived with a slightly different approach by T. M. Nieuwenhuizen, J. M. Luck, J. Canisius, J. L. von Hemmen, and W. J. Ventovogel. In: Special frequencies and Lifshitz singularities in binary random harmonic chains. Preprint Saclay PhT/85-183, submitted to J. Stat. Phys. 
